OPEN ACCESS

Edited by: Michael Kaess,

University of Bern, Switzerland

Reviewed by:

Ulrich W. Ebner-Priemer, Karlsruhe Institute of Technology (KIT),

Germany

Julian Koenig,

Heidelberg University, Germany

${ }^{*}$ Correspondence:

Glenn Kiekens

Glenn.Kiekens@kuleuven.be

Specialty section: This article was submitted to Mood and Anxiety Disorders,

a section of the journal

Frontiers in Psychiatry

Received: 30 July 2019 Accepted: 03 March 2020 Published: 20 March 2020

Citation:

Kiekens G, Hasking P, Nock MK, Boyes M, Kirtley $O$, Bruffaerts $R$, Myin-Germeys I and Claes L (2020) Fluctuations in Affective States and Self-Efficacy to Resist Non-Suicidal Self-Injury as Real-Time Predictors of Non-Suicidal Self-Injurious

Thoughts and Behaviors.

Front. Psychiatry 11:214. doi: 10.3389/fpsyt.2020.00214

\section{Fluctuations in Affective States and Self-Efficacy to Resist Non-Suicidal Self-Injury as Real-Time Predictors of Non-Suicidal Self-Injurious Thoughts and Behaviors}

\author{
Glenn Kiekens ${ }^{1,2 *}$, Penelope Hasking ${ }^{1}$, Matthew K. Nock ${ }^{3}$, Mark Boyes ${ }^{1}$, Olivia Kirtley ${ }^{4}$, \\ Ronny Bruffaerts ${ }^{2,5}$, Inez Myin-Germeys ${ }^{4}$ and Laurence Claes ${ }^{6,7}$ \\ ${ }^{1}$ School of Psychology, Curtin University, Perth, WA, Australia, ${ }^{2}$ Department of Neurosciences, Center for Public Health \\ Psychiatry, Leuven, Belgium, ${ }^{3}$ Department of Psychology, Harvard University, Cambridge, MA, United States, ${ }^{4}$ Department \\ of Neurosciences, Center for Contextual Psychiatry, KU Leuven, Leuven, Belgium, 5 Institute for Social Research, Population \\ Studies Center, University of Michigan, Ann Arbor, MI, United States, ${ }^{6}$ Faculty of Psychology and Educational Sciences, KU \\ Leuven, Leuven, Belgium, ${ }^{7}$ Faculty of Medicine and Health Sciences (CAPRI), University of Antwerp, Antwerp, Belgium
}

Introduction: Although research over the past decade has resulted in significantly increased knowledge about distal risk factors for non-suicidal self-injury (NSSI), little is known about short-term (proximal) factors that predict NSSI thoughts and behaviors. Drawing on contemporaneous theories of $\mathrm{NSSI}$, as well as the concept of ideation-to-action, the present study clarifies (a) real-time factors that predict NSSI thoughts and (b) the extent to which theoretically important momentary factors (i.e., negative affect, positive affect, and selfefficacy to resist NSSI) predict NSSI behavior in daily life, beyond NSSI thoughts.

Methods: Using experience sampling methodology, intensive longitudinal data was obtained from 30 young adults with frequent NSSI episodes in the last year. Participants completed assessments up to eight times per day for 12 consecutive days (signal-contingent sampling). This resulted in the collection of 2,222 assessments (median compliance $=79.2 \%$ ) during which 591 NSSI thoughts and $270 \mathrm{NSSI}$ behaviors were recorded. Using the dynamic structural equation modeling framework, multilevel vector autoregressive models were constructed.

Results: Within the same assessment, negative affect was positively associated with NSSI thoughts, whereas positive affect and self-efficacy to resist NSSI were each negatively associated with NSSI thoughts. Across assessments, higher-than-usual negative affect and self-efficacy to resist NSSI were predictive of short-term change in NSSI thoughts. While fluctuations in both negative affect and positive affect prospectively predicted NSSI behavior, these factors became non-significant in models that controlled for the predictive effect of NSSI thoughts. In contrast, self-efficacy to resist NSSI incrementally predicted a lower probability of engaging in NSSI, above and beyond NSSI thoughts. 
Discussion: This study provides preliminary evidence that affective fluctuations may uniquely predict NSSI thoughts but not NSSI behaviors, and point to the role of personal belief in the ability to resist NSSI in preventing NSSI behavior. These findings illustrate the need to differentiate between the development of NSSI thoughts and the progression from NSSI thoughts to behavior, as these are likely distinct processes, with different predictors.

Keywords: non-suicidal self-injury, real-time prediction, ideation-to-action, intensive longitudinal assessment, ecological momentary assessment

\section{INTRODUCTION}

Non-suicidal self-injury (NSSI), defined as the deliberate, selfinflicted damage of one's own body tissue without suicidal intent (e.g., cutting, scratching, and hitting oneself), is a worrisome behavior among adolescents and emerging adults $(1,2)$. Pooled lifetime prevalence estimates of NSSI are close to $17 \%-18 \%$ among adolescents and $12 \%-20 \%$ among emerging adults (3, 4). NSSI behaviors are an important predictor of future suicidal thoughts and behaviors (5-8) and psychopathology $(9,10)$, and are associated with stigma and feelings of shame (11-13), low levels of disclosure and help-seeking (14-16), and other adverse outcomes [e.g., poorer academic performance; (17)]. Together, these findings underscore the importance of a good understanding of the factors that underlie NSSI, with a view to informing preventive and intervention initiatives.

\section{The Short-Term Prediction Problem in Existing Research on NSSI}

While NSSI and its correlates have traditionally been studied using cross-sectional designs, over the past decade, concerted efforts have been made to clarify long-term (distal) predictors (18-21). These longitudinal studies typically take a populationlevel nomothetic approach (i.e., risk stratification at the betweenperson level), involving few measurement occasions (usually 25) that are spaced over long observation windows (e.g., yearly). Although such an approach may be useful in revealing whowithin the entire population-is at relatively high risk of engaging in NSSI in the next months or years, it lacks temporal resolution to reliably tell us when individuals at high risk are most likely to contemplate, or engage in, NSSI in the next minutes and hours. Providing greater clarity regarding shortterm (proximal) predictors requires a specific research design that takes an individualized ideographic approach (i.e., risk stratification at the within-person level) as well as intensive monitoring to capture momentary processes that explain imminent risk of NSSI. Fortunately, the recent proliferation of new technologies and smartphone-based apps have now made it feasible to use experience sampling methods to study NSSI and its real-time predictors in daily life (22).

\section{Affective Disturbances and NSSI}

A central focus of most theoretical models is that NSSI most often serves an affect regulation function (23-26). Empirical work supports that affect regulation is the most common reported reason for NSSI (27), and, consequently, many studies have focused on the predictive value of affective traits at the between-person level (28). This work revealed that both higher trait negative affect (i.e., tendency to experience more negative emotions) and lower trait positive affect (i.e., tendency to experience less positive emotions) are associated with risk of lifetime (29-31) and future NSSI behavior $(32,33)$. In line with this, emotional problems (especially anxiety and depressive symptoms) have been identified as risk factors of NSSI at the between-person level $(18,19,34)$. Unfortunately, fewer studies have focused on the role of affect in determining short-term risk for NSSI at the within-person level [for an overview see (35)]. One consistent finding across studies is that negative affect increases prior to NSSI (36-38), and predicts a higher probability of NSSI in the next hours (39-42). For instance, using ecological momentary assessment, Kranzler and colleagues observed that a momentary increase in negative affect positively predicted NSSI in the following 2-3 hours for adolescents and young adults (40). Similarly, Houben and colleagues, demonstrated that higher-than-usual negative affect increased the likelihood of NSSI in the next 30-120 minutes among a sample of inpatients (39). While these findings are important and support the affect regulation function of NSSI, future work is required beyond these initial studies to clarify the specificity of affective fluctuations in the short-term prediction of NSSI at the within-person level.

Of note, more research is warranted investigating the potential utility of positive affect as a protective factor against NSSI, as evidence to date has yielded inconclusive results. While some researchers have observed a decrease in positive affect in the hours prior to engagement in $\operatorname{NSSI}(36,37)$, others failed to confirm such a time trend (38), and found that lower-than-usual positive affect is not prospectively predictive of NSSI $(39,40)$. It may be that momentary lowered positive affect is more tolerable than increased negative affect, and therefore less relevant in eliciting NSSI (40). In any case, better understanding the role of affect requires thorough examination of specific emotions (e.g., relaxed, stressed), as well as composite constructs (e.g., positive affect). Retrospective studies, for instance, have demonstrated that people who self-injure report increased levels of positive emotions low in arousal (e.g., satisfied, relaxed) as well as decreased levels of negative emotions high in arousal (e.g., anxious, stressed) from prior to post NSSI (43, 44). However, because these studies are susceptible to memory biases that may distort these findings, experience sampling studies are warranted to evaluate whether low-arousal positive emotions, and/or high-arousal negative emotions, are most 
relevant in predicting NSSI within the next few hours. Providing greater clarity regarding the specificity of affective states as shortterm predictors of NSSI would provide valuable information for the development of novel preventive interventions.

\section{Affective Disturbances Predictive of NSSI Thoughts or NSSI Behavior?}

Surprisingly little is known about the extent to which affective fluctuations predict NSSI behavior, beyond NSSI thoughts. Originating from studies on suicidal thoughts and behaviors (45-47), the ideation-to-action framework argues that the factors that lead people to contemplate about a behavior (i.e., in this case thoughts concerning suicide) may not necessarily be the same factors that govern whether people act on their thoughts (i.e., attempt suicide). In a similar vein, it may be equally important to differentiate between the process of developing NSSI thoughts and engaging in NSSI behavior. NSSI thoughts are an important near-term precursor of NSSI behavior $(41,42)$, and a growing body of evidence suggests that momentary increased negative affect and lowered positive affect are salient factors in predicting NSSI thoughts $(40,41,48)$. As such, similar to the observation that affective disturbances are robust predictors of suicide ideation but not attempt $(47,49-51)$, the possibility exists that affective fluctuations are relevant in predicting short-term change in NSSI thoughts but are not uniquely predictive of making the transition from NSSI thoughts to behavior. While emerging evidence suggests that fluctuations in positive affect might be more useful in predicting thoughts than behavior (40), it is currently unclear whether negative and positive affective states hold predictive value beyond NSSI thoughts in determining short-term risk of NSSI behavior. Addressing this important gap in knowledge requires that future experience sampling studies carefully consider NSSI thoughts when evaluating affective states in the prediction of NSSI behavior.

If affective fluctuations are more useful in explaining shortterm change in NSSI thoughts than in predicting the occurrence of NSSI behavior, an important question is whether we can identify momentary factors that provide added insight into whether someone will transition from NSSI thoughts to behavior. Contemporaneous models of NSSI have begun to incorporate cognitive processes in explaining when people are at heightened risk of engaging in NSSI $(23,26)$. According to the Cognitive-Emotional Model of NSSI (23), NSSI-related cognitions determine whether someone who is experiencing an aversive emotional situation will, or will not, engage in NSSI in the next minutes and hours. Specifically, this model postulates that personal belief in the ability to resist NSSI will be a unique protective factor against NSSI behavior. While findings confirm that people who engage in NSSI report lower self-efficacy to resist NSSI than peers who do not self-injure $(23,52,53)$, experience sampling studies are warranted to evaluate whether these beliefs have utility in determining risk of NSSI behavior.

\section{The Present Study}

We designed the present study to clarify the extent to which momentary fluctuations in affective states and self-efficacy to resist NSSI are real-time predictors of NSSI thoughts and behaviors. Specifically, there were two major objectives at the within-person level. The first main objective was to evaluate whether within-person fluctuations in negative affect, positive affect, and self-efficacy to resist NSSI predict NSSI thoughts within the same observation window (i.e., contemporaneous associations reflecting processes occurring in the moment; objective 1a in Figure 1), as well as from one observation window to the next (i.e., temporal associations reflecting processes that unfold within hours; objective $1 \mathrm{~b}$ in Figure 1). Based on existing knowledge $(40,41,48)$, we hypothesized that higher-than-usual negative affect, and lower-than-usual positive affect, would each be contemporaneously and temporally associated with NSSI thoughts. However, as we expected that momentary fluctuations in affective states would trigger NSSI thoughts more strongly within minutes than hours, stronger effects were anticipated in contemporaneous than temporal models (54).

The second main objective was to evaluate whether withinperson variation in affective states and self-efficacy to resist NSSI, relative to their own average levels, predict NSSI behavior above and beyond NSSI thoughts (Figure 2). Building upon previous research from the suicide literature $(47,49,50)$, we hypothesized that fluctuations in affective states would not further increase the risk for NSSI behavior, after accounting for NSSI thoughts. To explore the utility of specific emotions, results were also analyzed using emotions as units of analyses rather than composite measures of negative and positive affect. As suggested by the Cognitive-Emotion Model of NSSI (23), we expected that self-efficacy to resist NSSI would negatively predict the occurrence of NSSI behavior above and beyond NSSI thoughts. Finally, in keeping with empirical work at the between-person level (18, 19, 32-34), an additional aim of the study was to evaluate trait negative affect, trait positive affect, self-efficacy to resist NSSI, and anxiety and depressive symptoms assessed at baseline as prospective predictors of NSSI thoughts and behaviors (Objective 3 ). Consistent with previous research and the ideation-to-action framework $(18,19,47,49,50)$, we hypothesized that depressive symptoms would uniquely predict mean-level of NSSI thoughts over time but not probability of NSSI behavior, whereas the opposite pattern of results was expected for selfefficacy to resist NSSI.

\section{METHODS}

\section{Participants and Procedure}

Participants were 30 young adults (Mean age $=20.1, \mathrm{SD}=1.1$; $80.0 \%$ female) selected from an ongoing longitudinal survey study of college students (19), meeting inclusion criteria of: (a) NSSI on 5 or more days in the last year, and (b) reported urges to self-injure in the past month. Participants were invited to the laboratory to complete self-report questionnaires and a diagnostic interview, and to receive training on completing the experience sampling protocol via "ExpiWell," a widely used smartphone app for 


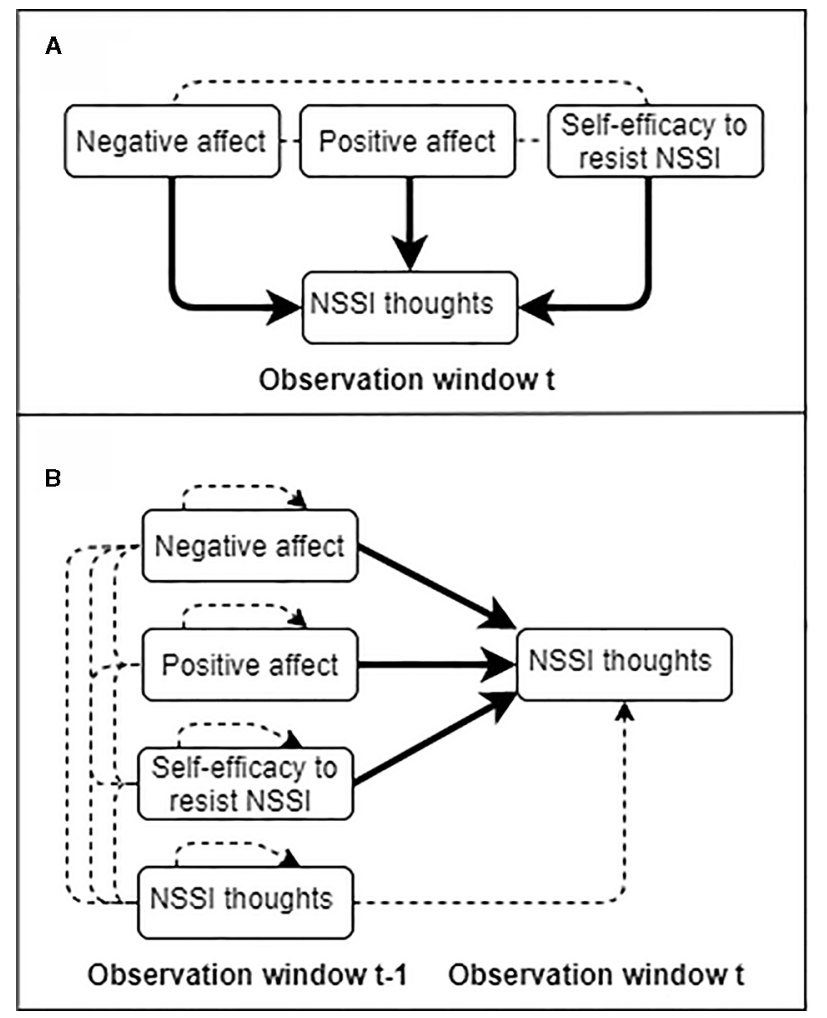

FIGURE 1 | Graphical illustration of the first research objective of the study in which NSSI thoughts are predicted within (Objective 1a depicted in panel A) and across measurement windows (Objective 1b depicted in panel B) at the within-person level. Solid arrows indicate effects of interest. Dotted arrows represent autoregressive effects and dotted lines associations within the same observation window.

real-time, and real-world, data collection (https://app.expiwell.com). The presence of 12-month mental disorders was assessed by means of the Structured Clinical Interview for DSM-5 (55). A clinical psychologist administered the following sections: mood disorders, anxiety disorders, obsessive-compulsive and related-disorders, substance use disorders, externalizing disorders, post-traumatic stress-disorder, and eating disorders. NSSI characteristics and suicidal thoughts and behaviors were assessed with the SelfInjurious Thoughts and Behaviors Interview (56). Table 1 presents an overview of the diagnostic features of the sample. Participants met diagnostic criteria for a median of two mental disorders in the last 12 months, with DSM-5 anxiety and mood disorders being most prevalent (range 33.3\%-50\%; Table 1). Participants reported a median of NSSI on 17.5 days in the past year (range 5-360 days), and used a median of 5 NSSI methods (ranging between 2 and 10). Two thirds of the sample (66.7\%) also reported having suicidal thoughts in the preceding 12 months, and $20 \%$ reported having made at least one suicide attempt in their life (median $=2.5$ attempts).

Following initial screening, participants were enrolled in a 12day signal-contingent experience sampling protocol in which they were prompted randomly eight times per day, between 9 a.m. and 9 p.m., in blocks of 1.5-h segments (minimum 15 minutes apart from prior assessments). Participants without a smartphone were provided with a personal device by the research team. To ensure that we captured people in their ongoing activities, and to avoid retrospective reporting, participants were required to register their response within 15 minutes of each prompt. Reimbursement for participation was structured to encourage compliance (42), with a financial compensation of $€ 25$ if compliance ranged between $25 \%$ and $50 \%$, €50 between $50 \%$ and $85 \%$, and $€ 75$ if compliance exceeded $85 \%$. Overall compliance was good (median $=79.2 \%$ ) with, on average, 74 randomly registered recordings per participant (range 36-95), resulting in 2,222 randomly registered recordings for the entire sample. Prior to enrollment, all participants were briefed about the procedures and the voluntary and confidential nature of the study and were provided with contact details of responsible clinicians (including the first and last author, both clinical psychologists). One item of the experience sampling protocol also assessed suicide ideation and activated a popup screen within the app with additional resources when participants reported experiencing suicidal thoughts. Written informed consent was provided by all participants and the study's protocol was approved by the University's Ethical Review Board and by the Belgian commission for the protection of privacy. All procedures were in accordance with the 1964 Helsinki declaration and its later amendments.

\section{Laboratory Measures}

Trait Positive and Negative Affect was assessed using the Positive and Negative Affect Schedule [PANAS; (57)]. The PANAS presents 10 positive (e.g., excited, attentive) and 10 negative emotions (e.g., distressed, nervous), and participants were asked to rate the extent to which they "generally" experience each emotion on a five-point Likert scale ranging from 1 (very slightly or not at all) to 5 (extremely). The PANAS is a reliable and valid measure that is invariant across demographic variables (58), and the internal consistency coefficients of both scales were also good in the current sample $\left(\alpha_{\mathrm{PA}}=0.78, \alpha_{\mathrm{NA}}=0.88\right)$.

Self-Efficacy to Resist NSSI was assessed using the six-item measure adapted from the Self-Efficacy to Avoid Suicidal Action Scale (59). In this study, participants reported from 1 (very uncertain) to 10 (very certain) whether they believe they can resist engaging in NSSI in the next 2 weeks (e.g., "How certain are you that you will not self-injure in the next two weeks?"), with higher scores indicating a higher personal belief in the ability to resist NSSI. The adapted NSSI version has shown good internal consistency coefficients in previous research $(52,53)$ as well as the current sample $(\alpha=0.74)$.

Anxiety and Depressive Symptoms in the past week were measured using the 21-item Depression Anxiety Stress Scales (60). The anxiety (e.g., "I felt I scared without any good reason") and depression (e.g., "I felt down-hearted and blue") scales include seven 4-point items ranging from 1 (Did not apply to me at all) to 4 (Applied to me very much or most of the time) that assess symptoms in the preceding week. Both scales have 


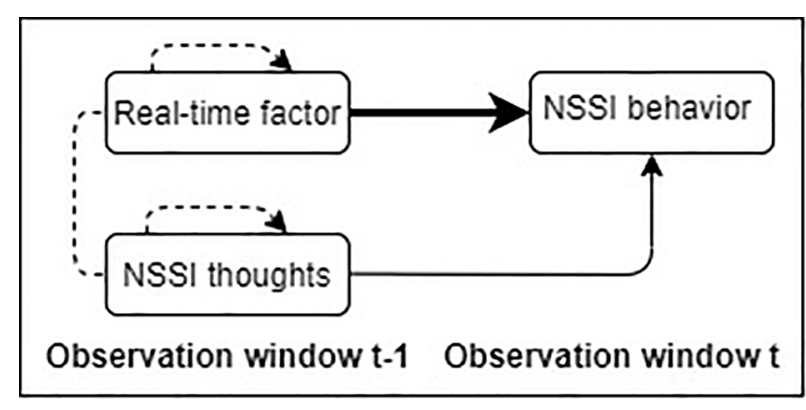

FIGURE 2 | Graphical illustration of the second research objective of the study in which NSSI behavior in daily life is predicted by real-time factors (i.e., negative affect, positive affect, and self-efficacy to resist NSSI), beyond NSSI thoughts at the within-person level. Solid arrows indicate effects of interest. Dotted arrows represent autoregressive effects and dotted lines associations within the same observation window.

demonstrated good internal consistency and convergent and discriminant validity in previous research (60). The internal consistency of the scales in the current sample was acceptable

TABLE 1 | Diagnostic characteristics of the sample $(n=30)$.

\begin{tabular}{|c|c|}
\hline & $\begin{array}{l}\% / \text { Median } \\
\text { (min; max) }\end{array}$ \\
\hline Mood disorders & 33.3 \\
\hline Major depressive disorder & 26.7 \\
\hline Persistent depressive disorder & 20.0 \\
\hline Anxiety disorders & 50.0 \\
\hline Panic disorder & 13.3 \\
\hline Agoraphobia & 6.7 \\
\hline Specific phobia & 16.7 \\
\hline Social anxiety disorder & 20.0 \\
\hline Generalized anxiety disorder & 23.3 \\
\hline Obsessive-compulsive disorders & 16.7 \\
\hline Alcohol use disorder (mild-moderate) & 13.3 \\
\hline ADHD & 6.7 \\
\hline Posttraumatic stress disorder & 13.3 \\
\hline Eating disorders & 20.0 \\
\hline Anorexia nervosa & 13.3 \\
\hline Bulimia nervosa & 3.3 \\
\hline Binge-eating disorder & 3.3 \\
\hline Any current mental disorder & 70.0 \\
\hline Number of current disorders & $2.0(0 ; 7)$ \\
\hline Number of days NSSI past year & $17.5(5 ; 360)$ \\
\hline Number of acts past month & $2.0(0 ; 60)$ \\
\hline Number of methods & $5(2 ; 10)$ \\
\hline \multicolumn{2}{|l|}{ Top 3 reported NSSI behaviors } \\
\hline Scratched oneself & 86.7 \\
\hline Cut oneself & 66.7 \\
\hline Hit hand/foot against wall/other objects & 56.7 \\
\hline Medically treated for NSSI & 26.7 \\
\hline \multicolumn{2}{|l|}{ Suicidal thoughts and behaviors } \\
\hline 12-month suicide ideation & 66.7 \\
\hline Lifetime suicide attempt & 20.0 \\
\hline
\end{tabular}

Mental disorders were defined as having met diagnostic criteria within the past year, with the exception of generalized anxiety disorder and ADHD which were defined as having met diagnostic criteria within the past 6 months.

$A D H D$, attention-deficit/hyperactivity disorder; NSSI, non-suicidal self-injury. for the Anxiety scale $(\alpha=0.61)$ and good for the Depressive scale $(\alpha=0.89)$.

\section{Ecological Momentary Assessment}

Momentary Positive and Negative Affect was assessed by asking respondents at each prompt to what extent they currently experience four positive (i.e., excited, cheerful, satisfied, relaxed), and six negative emotions (i.e., stressed, irritated, anxious, sad, hopeless, insecure): "Right now, I feel [emotion]." These specific emotions were selected because they represent a conceptual range of emotions within all quadrants of the affective circumplex defined by the dimensions of valence and arousal (61). Each emotion was rated on a 7-point Likert scale ranging from 0 (not at all) to 6 (very much), with the order in which emotions were presented randomized within persons, across beeps. Each affective state was calculated as a weighted mean across items. Using methods described by Shrout and Lane (62), both scales demonstrated excellent between-person reliability $\left(\mathrm{RKR}_{\mathrm{PA}}=0.98, \mathrm{RKR}_{\mathrm{NA}}=0.99\right)$, and good within-person reliability $\left(\mathrm{RC}_{\mathrm{PA}}=0.83, \mathrm{RC}_{\mathrm{NA}}=0.77\right)$.

\section{Momentary NSSI Thoughts and Occurrence of NSSI Behavior}

At each prompt, participants were asked to indicate whether they were currently thinking of engaging in NSSI ("Right now, I think about self-injuring without suicidal intent") using a 7-point Likert scale ranging from 0 (not at all) to 6 (a lot). Additionally, participants were asked to indicate whether or not they engaged in NSSI since their last registration ("Have you self-injured without wanting to die since the last beep?"). If answered affirmatively, a list of NSSI behaviors was presented including cutting/carving, scratching, hitting, burning, biting, head-banging, wound interfering, and an "other" category.

Momentary Self-Efficacy to Resist NSSI was measured by asking participants how confident they felt in their ability to resist NSSI ("How confident are you that you will not engage in NSSI till the next beep") using a 7-point Likert scale ranging from 0 (not confident at all) to 6 (very much confident).

\section{Statistical Analyses}

To accommodate the two-level structure of the data (i.e., observations nested within persons), and to provide understanding of the value of real-time predictors of NSSI thoughts and behavior, multilevel vector autoregressive models were constructed within the Dynamic Structural Equation Modeling Framework (DSEM) in Mplus $8.3(63,64)$. Contemporaneous associations between factors of interest and NSSI thoughts within the same window of measurement (Objective 1a) were examined using Residual DSEM, which is closely related to the regular DSEM framework, but allows modeling of the autoregressive part of the model while preserving the structural part on the contemporaneous relationships (65). Temporal relationships between factors of interest and both NSSI thoughts and behavior (Objectives $1 \mathrm{~b}$ and 2) were examined using regular DSEM. This allowed us to investigate the extent to which time-varying variables at $t-1$ 
(e.g., negative affect) predict NSSI thoughts and NSSI behavior at $t$, above and beyond the lagged version of the outcome variable (i.e., the autoregressive parameter) and/or a confound variable at $t-1$ (e.g., NSSI thoughts in the prediction of NSSI behavior). Latent person-mean centering was used to allow interpretation of predictor variables at the within-person level in a relative fashion for each person while accounting for sampling error. At the between-person level, we considered trait negative affect, trait positive affect, baseline self-efficacy, and anxiety and depressive symptoms in the past week as prospective predictors of NSSI thoughts and NSSI behaviors during the 12-day experience sampling protocol (Objective 3). These between-person variables were grand-mean centered to allow interpretation relative to the overall sample mean.

In all models, we used Bayesian estimation based on Markov Chain Monte Carlo using Gibbs sampling. Bayesian estimation has several advantages over a frequentist approach in this context, such as better performance in small samples (i.e., posterior distributions are not required to have asymptotically normal distributions). Non-informative priors were used in all analyses. Point estimates were obtained by taking the median of the posterior distributions for each parameter. Statistical significance was determined by estimating a 95\% credibility interval (CI) around each point estimate. A 1-hour transformed time interval was specified using the "TINTERVAL" statement to account for unequally-spaced intervals due to missing data and random sampling within blocks. This procedure creates a new time variable (measured in hours since first assessment in this study) and inserts based on the defined metric missing data records when no observation is present [for a detailed overview of this approach see (63)]. Missing data in DSEM is handled using a Kalman filter approach. Due to this procedure, all observations can be used in the analyses and a constant interpretation of lagged relations is maintained (66). Given that treating covariates as exogenous variables in time-series settings may yield biased estimates (65), autoregressive effects of covariates were included in both RDSEM and DSEM models. Bayesian linear regressions were used to predict continuous variables, such as NSSI thoughts, whereas Bayesian probit regression was used to predict the occurrence of NSSI behavior, which was modeled as a categorical variable (present/absent). Each model was specified using random intercepts with all other within-level parameters fixed, and was estimated using a minimum of 2,500 iterations with a thinning parameter of 20. Model convergence was ensured by checking that the potential scale reduction was close to 1 and trace plots did not contain trends, spikes, or other irregularities.

\section{RESULTS}

\section{Preliminary Descriptive and Variability Analyses}

During the 12-day experience sampling protocol, 591 NSSI thoughts (i.e., score higher than 0 ; mean intensity $=0.72$; $\mathrm{SD}=$ 1.48) were reported. Among those reporting NSSI thoughts
$(90 \%)$, on average $21.9(\mathrm{SD}=21.4 ;$ median $=16.0 ;$ range $1-70)$ NSSI thoughts were reported. Of the sample, $53.3 \%$ of the participants engaged in NSSI, with an average of 10.4 episodes during the 12-day experience sampling protocol $(\mathrm{SD}=10.7$; median $=6.0$; range 1-37). In total, 270 NSSI behaviors were recorded across 167 assessments (7.5\% of all sampled time points). Table 2 presents the descriptive and variability statistics for all within and between-person variables. These findings show that approximately half of the variability in negative affect and NSSI thoughts is due to within-person variance (vs. between person-variance). Figure 3 illustrates the within-person variability of NSSI thoughts on an hourly basis for participants. Although self-efficacy to resist NSSI varied more between than within individuals, positive affect showed considerably more variation at the within-person level.

\section{Contemporaneous Associations Between Affect, Self-Efficacy to Resist NSSI, and NSSI Thoughts (Objective 1a)}

We first investigated how variables were contemporaneously associated within the same time frame after partialing out temporal associations (Table 3). In univariate analyses, negative affect was significantly positively associated with NSSI thoughts, whereas positive affect and self-efficacy to resist NSSI were each negatively associated with NSSI thoughts. After controlling for shared variance within a multivariate modeling framework, each factor remained significantly associated, although weaker, with NSSI thoughts. Together, these contemporaneous associations explained $41 \%$ of the withinperson variance of NSSI thoughts in this sample.

\section{Fluctuations in Affective States and Self- Efficacy to Resist NSSI as Real-Time Predictors of NSSI Thoughts (Objective 1b)}

We constructed temporal models to determine the utility of negative affect, positive affect, and self-efficacy to resist NSSI as predictors of short-term change in NSSI thoughts (Table 4). Higher-than-usual negative affect at $t-1$ positively predicted NSSI thoughts at $t$, whereas higher-than-usual positive affect and self-efficacy to resist NSSI at $t-1$ negatively predicted NSSI thoughts at $t$. In a next step, we evaluated the unique value of these factors in a multivariate prediction model that simultaneously included all cross-regressive parameters at $t-$ 1. As can be seen in Table 4, higher-than-usual positive affect at $t-1$ became non-significant in predicting short-term change in NSSI thoughts at $t$. Fluctuations in negative affect and selfefficacy to resist NSSI at $t-1$ remained significantly predictive of short-term change in NSSI thoughts at $t$. Together, these temporal associations explained $18 \%$ of the variance of NSSI thoughts within persons, across time.

\section{Fluctuations in Affective States and Self- Efficacy to Resist NSSI as Real-Time Predictors of NSSI Behavior (Objective 2)}

To investigate the utility of fluctuations in affective states and self-efficacy to resist NSSI in predicting the occurrence of NSSI 
behavior beyond NSSI thoughts, we compared temporal models that included the autoregressive parameter of NSSI behavior to models that controlled the cross-regressive parameter of NSSI thoughts (Table 5). As expected, a very similar pattern of results as above was observed in models that included the lagged version of NSSI behavior. Higher-than-usual negative affect at $t-1$ was significantly positively predictive of the occurrence of NSSI behavior, whereas higher-than-usual positive affect and selfefficacy to resist NSSI at $t-1$ were significantly negatively predictive of NSSI behavior in the next time interval. However, when we controlled the cross-regressive parameter of NSSI thoughts at $t-1$, fluctuations in negative affect and positive affect at $t-1$ both became non-significant predictors of NSSI behavior at $t$. In contrast, higher-than-usual belief in one's ability to resist NSSI at $t-1$ remained significantly predictive of a lower probability of NSSI behavior at $t$.

Next, we evaluated whether specific emotions, rather than affective composite scores, hold incremental predictive value in predicting NSSI behavior (Table 6). All assessed negative emotions at $t-1$ (except for feeling irritated) were predictive of NSSI behavior at $t$ in models including the lagged version of NSSI behavior. However, when controlling the cross-regressive parameter of NSSI thoughts at $t-1$, again, all negative emotions at $t-1$ became non-significant in predicting NSSI behavior at $t$. Conversely, all assessed positive emotions at $t-1$ were consistently negatively predictive of NSSI behavior at $t$ in models including the lagged version of NSSI behavior at $t-1$. However when controlling the cross-regressive parameter of NSSI thoughts at $t-1$, the feeling "relaxed" at $t-1$ remained negatively predictive of NSSI behavior at $t$.

\section{Trait Affect, Self-Efficacy to Resist NSSI, and Anxiety and Depressive Symptoms as Predictors of NSSI Thoughts and NSSI Behaviors (Objective 3)}

Finally, we examined the utility of baseline measures of trait affect, self-efficacy to resist NSSI, and past-week anxiety and depressive symptoms as between-person predictors of NSSI thoughts and NSSI behavior (Supplementary Materials). This revealed that individuals with lower mean scores on trait positive affect $(\beta=-0.09,95 \% \mathrm{CI}=-0.16 ;-0.02)$ and higher mean scores on past week depressive symptoms $(\beta=0.11,95 \% \mathrm{CI}=0.04$; 0.18 ) reported higher mean levels of NSSI thoughts across the 12day experience sampling protocol. Yet, only depressive symptoms uniquely predicted a higher mean level of NSSI thoughts across time $(\beta=0.11,95 \% \mathrm{CI}=0.01 ; 0.22)$. In contrast, self-efficacy to resist NSSI at baseline was the only factor that significantly predicted engagement in NSSI behavior during the 12 -day experience sampling protocol $(\beta=-0.06,95 \%$ $\mathrm{CI}=-0.15 ;-0.00)$.

\section{DISCUSSION}

Obtaining a better understanding of the factors that determine when individuals at high risk are most likely to contemplate, or engage in, NSSI behavior represents a challenging but critical research frontier $(35,67)$. To this end, using smartphone-based assessment of young adults who frequently self-injure, the present study provides a preliminary investigation into the extent to which affective states and self-efficacy to resist NSSI are real-time predictors of NSSI thoughts and behaviors. To our knowledge, this is the first experience sampling study to differentiate between the process of experiencing NSSI thoughts and engaging in NSSI behavior. Results suggest that affective fluctuations (especially negative affect) may be more useful in predicting NSSI thoughts than behavior per se, and point to the role of cognitive factors (i.e., belief in one's ability to resist NSSI) in preventing NSSI behavior among people experiencing NSSI thoughts.

NSSI thoughts varied considerably across hours, illustrating the need for intensive monitoring to capture these fluctuations in daily life. The first aim of the study was to identify real-time factors that explain variability in NSSI thoughts. Consistent with previous work $(40,41,48)$, higher-than-usual negative affect cooccurred with NSSI thoughts and uniquely predicted a stronger

TABLE 2 | Descriptive and variability statistics of negative affect, positive affect, self-efficacy to resist NSSI, and NSSI thoughts and behaviors during 12-day experience sampling protocol.

\begin{tabular}{|c|c|c|c|c|c|c|}
\hline Within-person variables & $\mathbf{M} / \mathbf{N}$ & $\mathrm{SD} / \%$ & Range & Total variance $^{a}$ & $I^{\prime C C} C^{b}$ & $95 \% \mathrm{Cl}$ \\
\hline NSSI thoughts & 0.72 & 1.48 & $0-6$ & 2.38 & .51 & $.38-.65$ \\
\hline Negative affect & 1.74 & 1.19 & $0-6$ & 1.52 & .46 & $.34-.61$ \\
\hline Positive affect & 2.94 & 1.28 & $0-6$ & 1.73 & .33 & $.23-.47$ \\
\hline Self-efficacy to resist NSSI next hours & 4.79 & 1.74 & $0-6$ & 3.34 & .70 & $.59-.80$ \\
\hline Number of assessments NSSI behavior reported & 167 & 7.52 & $0-1$ & - & - & - \\
\hline Between-person variables & $\mathbf{M}$ & SD & Range & - & - & - \\
\hline Trait negative affect & 29.33 & 7.88 & $5-50$ & & & \\
\hline Trait positive affect & 30.13 & 5.21 & $5-50$ & & & \\
\hline Self-efficacy to resist NSSI next 2 weeks & 31.93 & 10.73 & $6-60$ & & & \\
\hline Anxiety symptoms past week & 13.60 & 3.66 & $7-28$ & & & \\
\hline Depressive symptoms past week & 14.53 & 5.24 & $7-28$ & & & \\
\hline
\end{tabular}

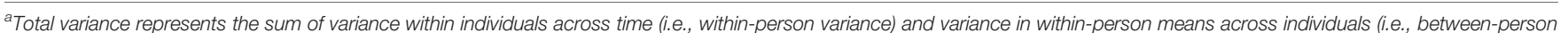
variance).

${ }^{b}$ The ICC represents the proportion of the total variance that is accounted for by between-person variance.

NSSI, non-suicidal self-injury; ICC, intraclass correlation; $95 \% \mathrm{Cl}$, credibility interval; $M$, mean; SD, standard deviation; $N$, total number. 


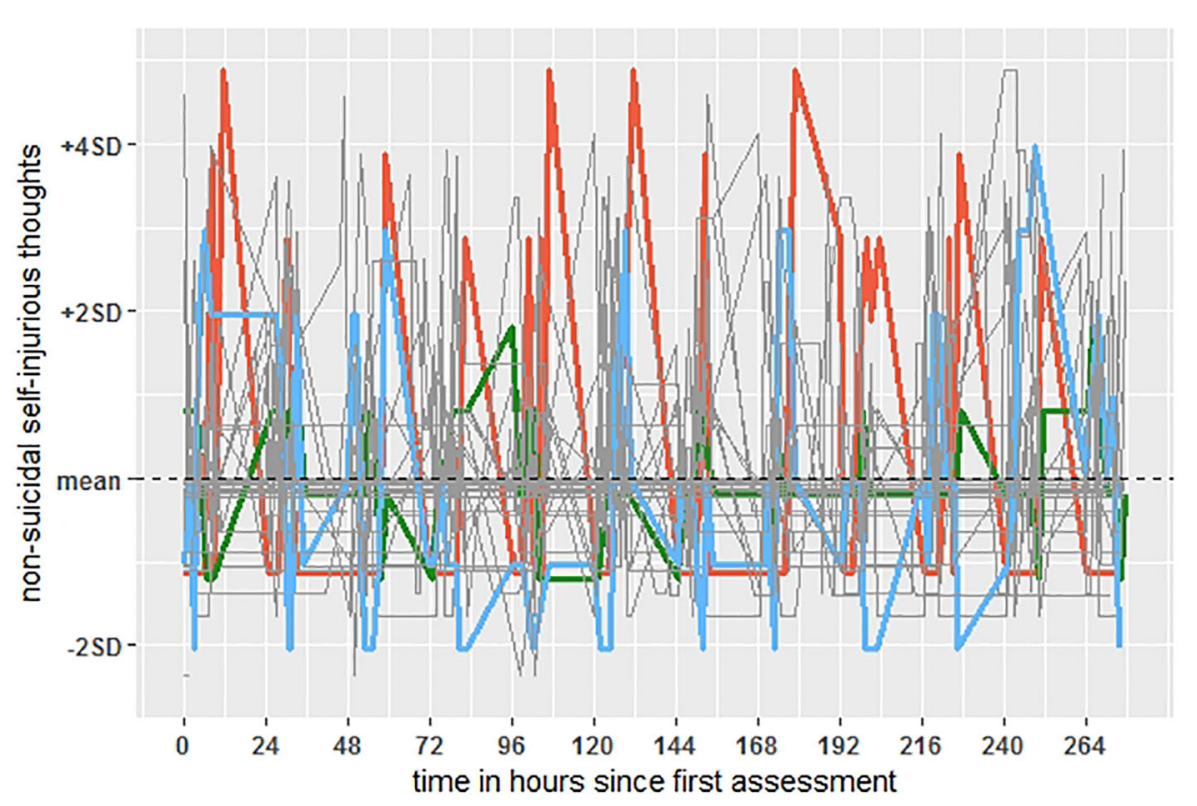

FIGURE 3 | Times series plot of non-suicidal self-injurious thoughts during the 12-day assessment period. Values are person-mean centered (comparing each's participant's hourly level of non-suicidal self-injurious thoughts to that individual's overall average across time; dashed line). The colored lines represent three randomly selected participants to illustrate within-person variability on an hourly basis.

TABLE 3 | Contemporaneous (moment-to-moment) associations between affective states, self-efficacy to resist NSSI, and non-suicidal self-injurious thoughts.

\begin{tabular}{|c|c|c|c|c|}
\hline & \multicolumn{2}{|c|}{ Univariate analyses $^{a}$} & \multicolumn{2}{|c|}{ Full multivariate analyses ${ }^{b}$} \\
\hline & B (SD) & $95 \% \mathrm{Cl}$ & B (SD) & $95 \% \mathrm{Cl}$ \\
\hline \multicolumn{5}{|c|}{ Contemporaneous within-person associations } \\
\hline Negative affect $t$ & $0.48(0.02)$ & $0.43 ; 0.53$ & $0.28(0.03)$ & $0.22 ; 0.33$ \\
\hline Positive affect $t$ & $-0.33(0.02)$ & $-0.37 ;-0.29$ & $-0.05(0.02)$ & $-0.09 ;-0.00$ \\
\hline Self-efficacy to resist NSSI $t$ & $-0.58(0.02)$ & $-0.61 ;-0.53$ & $-0.48(0.02)$ & $-0.52 ;-0.45$ \\
\hline
\end{tabular}

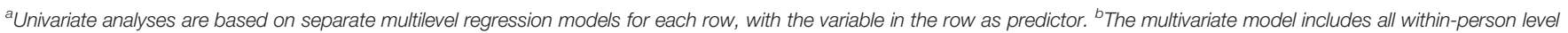
variables in one multilevel regression model (cf. Figure 1A).

$B$, median unstandardized point estimate; SD, posterior standard deviation; Cl, credibility interval.

Bolded cells indicate that there is a 95\% probability that the true population value is not null.

intensity of NSSI thoughts from one observation window to the next. The latter provides further evidence that increased negative affect is a robust short-term risk factor for NSSI thoughts. In contrast, while lower-than-usual positive affect was negatively associated with NSSI thoughts, this association did not transcend uniquely across time periods. There are two explanations for this: a) positive affect is only relevant in identifying NSSI thoughts as they occur, or b) positive affect also acts as a buffer against NSSI thoughts, but this protective effect occurs on a shorter timescale than the hourly scale used in this study. In line with the latter, we observed substantial within-person variability [intraclass correlation $(\mathrm{ICC})=0.33$ ] in positive affect from hour to hour. However, future research with even greater temporal resolution is needed to rule out one of these explanations. Finally, we found evidence that individuals were less likely to consider NSSI when they had high momentary belief in their ability to resist NSSI, which, in turn, prospectively predicted a lower intensity of NSSI thoughts one hour later.

Importantly, associations between affective states and NSSI thoughts were considerably weaker in temporal than contemporaneous models. Although the temporal precedence of associations cannot be determined in contemporaneous models (i.e., whether affect changes NSSI thoughts, or vice versa), researchers have advocated that contemporaneous relationships, which represent a snapshot in time, may uncover fast-moving causal processes (54). Given the time frame of measurement in this study, this likely suggests that the connection between momentary affect and the manifestation of NSSI thoughts is a fast occurring process that operates within seconds and minutes rather than hours. Again, this implies that better understanding the time frame of these relationships represents an important avenue for future research, as this will 
TABLE 4 | Temporal within-person associations between affective states, self-efficacy to resist NSSI, and non-suicidal self-injurious thoughts.

\begin{tabular}{|c|c|c|c|c|}
\hline & \multicolumn{2}{|c|}{$\begin{array}{l}\text { Analyses controlling } \\
\text { NSSI thoughts at } t-1 \mathrm{~h}^{\mathrm{a}}\end{array}$} & \multicolumn{2}{|c|}{ Full multivariate analyses ${ }^{b}$} \\
\hline & B (SD) & $95 \% \mathrm{Cl}$ & B (SD) & $95 \% \mathrm{Cl}$ \\
\hline \multicolumn{5}{|l|}{ Temporal within-person associations } \\
\hline NSSI thoughts $t-1 \mathrm{~h}$ & $0.47(0.02)$ & $0.43 ; 0.52$ & $0.26(0.03)$ & $0.20 ; 0.33$ \\
\hline Negative affect $t-1 \mathrm{~h}$ & $0.17(0.03)$ & $0.11 ; 0.23$ & $0.09(0.04)$ & $0.02 ; 0.17$ \\
\hline Positive affect $t-1 \mathrm{~h}$ & $-0.10(0.02)$ & $-0.15 ;-0.05$ & $0.00(0.03)$ & $-0.05 ; 0.06$ \\
\hline Self-efficacy to resist NSSI $t-1 \mathrm{~h}$ & $-0.23(0.03)$ & $-0.30 ;-0.17$ & $-0.18(0.03)$ & $-0.24 ;-0.12$ \\
\hline
\end{tabular}

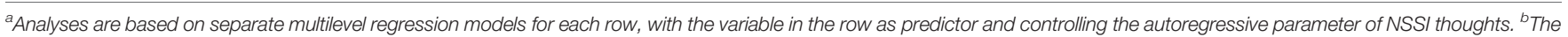
multivariate model includes all within-person variables in one multilevel regression model (cf. Figure 1B).

$B$, median unstandardized point estimate; SD, posterior standard deviation; Cl, credibility interval.

Bolded cells indicate that there is a $95 \%$ probability that the true population value is not null.

TABLE 5 | Temporal within-person associations between affective states, self-efficacy to resist NSSI, and non-suicidal self-injury.

\begin{tabular}{|c|c|c|c|c|}
\hline & \multicolumn{2}{|c|}{$\begin{array}{l}\text { Analyses controlling } \\
\text { NSSI behavior } t-1 \mathrm{~h}^{\mathrm{a}}\end{array}$} & \multicolumn{2}{|c|}{$\begin{array}{l}\text { Analyses controlling } \\
\text { NSSI thoughts } t-1 h^{b}\end{array}$} \\
\hline & B (SD) & $95 \% \mathrm{Cl}$ & B (SD) & $95 \% \mathrm{Cl}$ \\
\hline \multicolumn{5}{|l|}{ Temporal within-person associations } \\
\hline NSSI behavior $t-1 \mathrm{~h}$ & $0.31(0.10)$ & $0.07 ; 0.49$ & $0.04(0.14)$ & $-0.23 ; 0.28$ \\
\hline NSSI thoughts $t-1 \mathrm{~h}$ & $0.32(0.07)$ & $0.20 ; 0.45$ & $0.32(0.05)$ & $0.21 ; 0.43$ \\
\hline Negative affect $t-1 \mathrm{~h}$ & $0.26(0.08)$ & $0.12 ; 0.41$ & $0.14(0.10)$ & $-0.06 ; 0.33$ \\
\hline Positive affect $t-1 \mathrm{~h}$ & $-0.19(0.06)$ & $-0.32 ;-0.09$ & $-0.12(0.07)$ & $-0.26 ; 0.03$ \\
\hline Self-efficacy to resist NSSI $t-1 \mathrm{~h}$ & $-0.43(0.08)$ & $-0.58 ;-0.29$ & $-0.33(0.08)$ & $-0.49 ;-0.19$ \\
\hline
\end{tabular}

TABLE 6 | Temporal within-person associations between specific emotions and non-suicidal self-injury.

\begin{tabular}{|c|c|c|c|c|}
\hline \multirow[b]{2}{*}{ Temporal within-person associations } & \multicolumn{2}{|c|}{$\begin{array}{l}\text { Analyses controlling } \\
\text { NSSI behavior } t-1 \mathrm{~h}^{\mathrm{a}}\end{array}$} & \multicolumn{2}{|c|}{$\begin{array}{l}\text { Analyses controlling } \\
\text { NSSI thoughts } t-1 \mathrm{~h}^{\mathrm{b}}\end{array}$} \\
\hline & B (SD) & $95 \% \mathrm{Cl}$ & B (SD) & $95 \% \mathrm{Cl}$ \\
\hline \multicolumn{5}{|l|}{ Negative emotions high-arousal } \\
\hline Anxious $t-1 \mathrm{~h}$ & $0.21(0.07)$ & $0.08 ; 0.35$ & $0.10(0.08)$ & $-0.05 ; 0.27$ \\
\hline Irritated $t-1 \mathrm{~h}$ & $0.09(0.06)$ & $-0.03 ; 0.20$ & $0.06(0.06)$ & $-0.07 ; 0.18$ \\
\hline Stressed $t-1 \mathrm{~h}$ & $0.13(0.05)$ & $0.03 ; 0.23$ & $0.09(0.06)$ & $-0.02 ; 0.21$ \\
\hline Hopeless $t-1 \mathrm{~h}$ & $0.11(0.05)$ & $0.01 ; 0.21$ & $0.02(0.06)$ & $-0.11 ; 0.14$ \\
\hline Insecure $t-1 \mathrm{~h}$ & $0.17(0.07)$ & $0.05 ; 0.31$ & $0.08(0.07)$ & $-0.05 ; 0.22$ \\
\hline \multicolumn{5}{|l|}{ Positive emotions high-arousal } \\
\hline Cheerful $t-1 \mathrm{~h}$ & $-0.17(0.06)$ & $-0.28 ;-0.07$ & $-0.12(0.06)$ & $-0.24 ; 0.00$ \\
\hline Excited $t-1 \mathrm{~h}$ & $-0.13(0.05)$ & $-0.23 ;-0.04$ & $-0.05(0.06)$ & $-0.18 ; 0.07$ \\
\hline \multicolumn{5}{|l|}{ Positive emotions low-arousal } \\
\hline
\end{tabular}


provide unique insight into effects that may unfold across shorter and/or longer time intervals.

The second aim of the study was to evaluate the extent to which fluctuations in affective states and self-efficacy to resist NSSI predict NSSI behavior beyond the effect of NSSI thoughts. In line with previous work (39-41), we found that fluctuations in negative affect prospectively predicted NSSI behavior when NSSI thoughts were not accounted for. When accounting for NSSI thoughts, however, negative affect was no longer significantly predictive of NSSI behavior. Following an ideation-to-action framework (45-47), we do not believe this pattern of findings to indicate that negative affect is unimportant in the manifestation of NSSI behavior-indeed it leads people at high risk to more intensively contemplate engaging in NSSI-but only that it will not exert an additional effect beyond intensity of thoughts in determining whether someone will progress and engage in NSSI. We found similar findings for positive affect: higher-than usual positive affect was not uniquely predictive of a lower probability of engaging in NSSI behavior when taking into consideration NSSI thoughts. Further analyses showed similar findings for all but one emotion (i.e., feeling relaxed), which reflects-relative to feeling satisfied-an absence of arousal within the low positive valence quadrant $(61,68)$. Although caution is needed interpreting this finding, it suggests that focusing on the down-regulation of physiological hyper-arousal $(69,70)$, when thoughts of NSSI occur, may be one useful strategy to interrupt the transition to NSSI behavior. Taken together, these findings provide preliminary evidence that affective states may be unique real-time predictors of NSSI thoughts but not behavior.

If replicated, the implications are far-reaching as it would reflect the necessity of treating the development of thoughts and the subsequent transition from NSSI thoughts to behavior as separate processes that may come with separate sets of predictors. Making the distinction between NSSI thoughts/ behaviors may not only be important from a theoretical, but also from a clinical viewpoint. Researchers observed that it typically takes people who self-injure between 1 and 30 minutes to transition from thoughts to behavior $(42,71)$. This implies that, in most instances, there will be a brief window of opportunity to intervene and interrupt the transition to behavioral action. Ecological momentary interventions using mobile devices might have particular merit in this context (22, $72,73)$, as these can be delivered when people report experiencing NSSI thoughts, and facilitate relapse prevention techniques. In line with the Cognitive-Emotional Model of NSSI (23), we found evidence that low self-efficacy to resist NSSI may be particularly relevant in identifying high-risk situations among people experiencing NSSI thoughts.

The third aim of the study was to investigate population-level predictors at the between-person level. In line with findings in suicide research $(47,49,50)$ and the Cognitive-Emotional Model of NSSI (23), people with higher levels of depressive symptoms at baseline reported more intense thoughts over the course of the study, but only low self-efficacy to resist NSSI in the next two weeks explained who, in our student sample, engaged in NSSI.
This is consistent with the concept of capability for suicide (74), which specifies that a person must hold beliefs about their ability to self-injure (i.e., low self-efficacy to resist) in order to act on self-injurious thoughts. In sum, these findings provide novel evidence for the clinical utility of NSSI-related cognitions in determining relative risk of future NSSI behavior, and suggest that boosting self-efficacy to resist NSSI might be an important step in equipping people who self-injure with the confidence to handle high risk aversive emotional situations in everyday life.

\section{Limitations and Future Research Directions}

Several limitations should be considered in interpreting the findings of this study. First, and foremost, as this sample comprised 30 (mostly female) young adults, replication is warranted in larger samples including more males. Second, and relatedly, the generalizability of the findings to clinical samples is unclear and should be studied. It might be that clinical samples show stronger temporal relationships between affective states and NSSI thoughts and behaviors. The current findings should, therefore, be considered as preliminary. In fact, a major future research avenue will be to allow subject-specific effects (for which the current sample was too small) to clarify how these within-person relationships differ between people, as a function of person-level features, such as diagnostic status, gender, personality traits, and experienced life events. Third, all participants within the sample had already engaged in NSSI. This is in contrast to the majority of ideation-to-action research on suicidal thoughts and behaviors, where ideation is considered only in the absence of behavior and separate groups of individuals with ideation and those with behavior are compared. It is possible that factors predicting NSSI thoughts may differ between individuals who have and have not already engaged in NSSI behavior. Contemporary ideation-to-action models of suicide have not explicitly considered factors associated with ideation among individuals who have already engaged in suicidal behavior $(45,46,74,75)$. Consequently, a fruitful direction for future research could be to compare ideation-to-action pathways between those who have and have not already engaged in NSSI. Fourth, while the experience sampling protocol we implemented is among the most longitudinally intensive studies conducted thus far (assessments every 90 minutes), this did not allow us to track dynamic processes that happen in the moments that lead up to NSSI. To address this shortcoming, future experience sampling studies could incorporate burst assessments (i.e., multiple beeps over shorter time periods) when individuals report NSSI thoughts. Given that NSSI typically occurs within a narrow time frame following NSSI thoughts $(42,71)$, such studies would also provide a unique opportunity to clarify the immediate affective-cognitive consequences of engaging in NSSI.

Fifth, although experience sampling reduces recall bias, it still relies on self-report and the ability of participants to accurately describe their thoughts, feelings, and behaviors. Future studies may want to investigate if incorporating wearable devices that detect information about people's movement and activity and 
psychophysiology (e.g., electro-dermal activity and heartrate variability) could augment the short-term prediction of NSSI thoughts and behavior beyond self-report. Use of wearable technology for these purposes is already emerging in suicide research $(76,77)$. Sixth, although overall compliance was high, considering the intensive sampling protocol, on average participants failed to respond to one in five prompts, and it is unclear to what extent this may have impacted the results. Finally, to reduce participant burden, we decided to operationalize NSSI thoughts using a single item similar to previous studies (42). Building upon these findings, future studies may want to evaluate different qualitative aspects relating to NSSI thoughts [i.e., intensity, duration, controllability; $(71,78)]$, and explore whether meaningful patterns can be identified in relationship to risk for NSSI behavior. In suicide research, for instance, scholars have identified different phenotypes of suicidal thinking, and were able to associate a thought profile characterized by severe persistent suicidal thoughts to a recent suicide attempt (79).

\section{CONCLUSION}

The present study provides novel evidence that affective fluctuations may be more central to the prediction of NSSI thoughts than NSSI behavior, and suggests that perceiving oneself to be able to resist NSSI, might be key in determining risk of NSSI behavior among people experiencing NSSI thoughts. We believe these findings illustrate the merit of carefully delineating between the processes of developing thoughts and making the transition to behavior, and we hope it encourages researchers to further investigate the relative importance of momentary factors for the different stages towards engagement in NSSI.

\section{DATA AVAILABILITY STATEMENT}

The datasets generated for this study are available on request to the corresponding author.

\section{ETHICS STATEMENT}

The studies involving human participants were reviewed and approved by the Social and Societal Ethics Committee (KU

\section{REFERENCES}

1. International Society for the Study of Self-injury. What is self-injury? (2018). Available from: https://itriples.org/about-self-injury/what-is-self-injury/.

2. Gillies D, Christou MA, Dixon AC, Featherston OJ, Rapti I, Garcia-Anguita A, et al. Prevalence and Characteristics of Self-Harm in Adolescents: Meta-Analyses of Community-Based Studies 1990-2015. J Am Acad Child Adolesc Psychiatry (2018) 57:733-41. doi: 10.1016/j.jaac.2018.06.018

3. Muehlenkamp JJ, Claes L, Havertape L, Plener PL. International prevalence of adolescent non-suicidal self-injury and deliberate self-harm. Child Adolesc Psychiatry Ment Health (2012) 6:10. doi: 10.1186/1753-2000-6-10
Leuven). The patients/participants provided their written informed consent to participate in this study.

\section{AUTHOR CONTRIBUTIONS}

GK: study design, data collection, data analysis, interpretation of results, writing initial drafts of the manuscript, and critical revision for important intellectual content. PH: study design, interpretation of results, and critical revision for important intellectual content. $\mathrm{MN}$ : critical revision for important intellectual content. MB: study design and critical revision for important intellectual content. OK: study design and critical revision for important intellectual content. IM-G: study design and critical revision for important intellectual content. RB: critical revision for important intellectual content. LC: study design, data-collection, interpretation of results, critical revision for important intellectual content, and supervision of all aspects of the study. All authors approved the final version of the manuscript.

\section{FUNDING}

This research was supported by grants from the Research Foundation Flanders [1114717N (GK), 1114719N (GK)], and Curtin University [CIPRS/HSFIRS (GK)]. The funding sources had no role in the design and conduct of the study; collection, management, analysis, and interpretation of the data; preparation, review, or approval of the manuscript; and decision to submit the manuscript for publication.

\section{ACKNOWLEDGMENTS}

The authors wish to thank the individuals who participated in the intensive and demanding protocol of this study.

\section{SUPPLEMENTARY MATERIAL}

The Supplementary Material for this article can be found online at: https://www.frontiersin.org/articles/10.3389/fpsyt.2020.00214/ full\#supplementary-material

4. Swannell SV, Martin GE, Page A, Hasking P, St John NJ. Prevalence of nonsuicidal self-injury in nonclinical samples: systematic review, metaanalysis and meta-regression. Suicide Life Threat Behav (2014) 44:273-303. doi: $10.1111 /$ sltb. 12070

5. Kiekens G, Hasking P, Boyes M, Claes L, Mortier P, Auerbach RP, et al. The associations between non-suicidal self-injury and first onset suicidal thoughts and behaviors. J Affect Disord (2018) 239:171-9. doi: 10.1016/ j.jad.2018.06.033

6. Mortier P, Demyttenaere K, Auerbach RP, Cuijpers P, Green JG, Kiekens G, et al. First onset of suicidal thoughts and behaviours in college. J Affect Disord (2017) 207:291-9. doi: 10.1016/j.jad.2016.09.033 
7. Ribeiro JD, Franklin JC, Fox KR, Bentley KH, Kleiman EM, Chang BP, et al. Self-injurious thoughts and behaviors as risk factors for future suicide ideation, attempts, and death: a meta-analysis of longitudinal studies. Psychol Med (2016) 46:225-36. doi: 10.1017/S0033291715001804

8. Hamza CA, Willoughby T. Nonsuicidal Self-Injury and Suicidal Risk Among Emerging Adults. J Adolesc Health (2016) 59:411-5. doi: 10.1016/ j.jadohealth.2016.05.019

9. Wilkinson PO, Qiu T, Neufeld S, Jones PB, Goodyer IM. Sporadic and recurrent non-suicidal self-injury before age 14 and incident onset of psychiatric disorders by 17 years: prospective cohort study. Br J Psychiatry (2018) 212:222-6. doi: 10.1192/bjp.2017.45

10. Riley EN, Davis HA, Combs JL, Jordan CE, Smith GT. Nonsuicidal Self-injury as a Risk Factor for Purging Onset: Negatively Reinforced Behaviours that Reduce Emotional Distress. Eur Eat Disord Rev (2016) 24:78-82. doi: 10.1002/ erv. 2407

11. Burke TA, Piccirillo ML, Moore-Berg SL, Alloy LB, Heimberg RG. The stigmatization of nonsuicidal self-injury. J Clin Psychol (2019) 75:481-98. doi: $10.1002 /$ jclp. 22713

12. Mahtani S, Hasking P, Melvin GA. Shame and Non-suicidal Self-injury: Conceptualization and Preliminary Test of a Novel Developmental Model among Emerging Adults. J Youth Adolesc (2019) 48:753-70. doi: 10.1007/ s10964-018-0944-0

13. Hasking P, Boyes M. Cutting Words: A Commentary on Language and Stigma in the Context of Nonsuicidal Self-Injury. J Nerv Ment Dis (2018) 206:829-33. doi: 10.1097/NMD.0000000000000899

14. Gollust SE, Eisenberg D, Golberstein E. Prevalence and correlates of selfinjury among university students. J Am Coll Health (2008) 56:491-8. doi: 10.3200/jach.56.5.491-498

15. Whitlock J, Muehlenkamp J, Purington A, Eckenrode J, Barreira P, Baral Abrams G, et al. Nonsuicidal self-injury in a college population: general trends and sex differences. J Am Coll Health (2011) 59:691-8. doi: 10.1080/ 07448481.2010 .529626

16. Macrynikola N, Miranda R, Soffer A. Social connectedness, stressful life events, and self-injurious thoughts and behaviors among young adults. Compr Psychiatry (2018) 80:140-9. doi: 10.1016/j.comppsych.2017.09.008

17. Kiekens G, Claes L, Demyttenaere K, Auerbach RP, Green JG, Kessler RC, et al. Lifetime and 12-Month Nonsuicidal Self-Injury and Academic Performance in College Freshmen. Suicide Life Threat Behav (2016) 46:56376. doi: $10.1111 /$ sttb.12237

18. Fox KR, Franklin JC, Ribeiro JD, Kleiman EM, Bentley KH, Nock MK. Metaanalysis of risk factors for nonsuicidal self-injury. Clin Psychol Rev (2015) 42:156-67. doi: 10.1016/j.cpr.2015.09.002

19. Kiekens G, Hasking P, Claes L, Boyes M, Mortier P, Auerbach RP, et al. Predicting the incidence of non-suicidal self-injury in college students. Eur Psychiatry (2019) 59:44-51. doi: 10.1016/j.eurpsy.2019.04.002

20. Buelens T, Luyckx K, Gandhi A, Kiekens G, Claes L. Non-Suicidal Self-Injury in Adolescence: Longitudinal Associations with Psychological Distress and Rumination. J Abnormal Child Psychol (2019) 47:1569-81. doi: 10.1007/ s10802-019-00531-8

21. Tatnell R, Kelada L, Hasking P, Martin G. Longitudinal analysis of adolescent NSSI: the role of intrapersonal and interpersonal factors. J Abnorm Child Psychol (2014) 42:885-96. doi: 10.1007/s10802-013-9837-6

22. Myin-Germeys I, Kasanova Z, Vaessen T, Vachon H, Kirtley O, Viechtbauer W, et al. Experience sampling methodology in mental health research: new insights and technical developments. World Psychiatry (2018) 17:123-32. doi: 10.1002/wps.20513

23. Hasking P, Whitlock J, Voon D, Rose A. A cognitive-emotional model of NSSI: using emotion regulation and cognitive processes to explain why people self-injure. Cognit Emot (2017) 31:1543-56. doi: 10.1080/02699931.2016.1241219

24. Nock MK, Prinstein MJ. A functional approach to the assessment of selfmutilative behavior. J Consult Clin Psychol (2004) 72:885-90. doi: 10.1037/ 0022-006X.72.5.885

25. Chapman AL, Gratz KL, Brown MZ. Solving the puzzle of deliberate selfharm: the experiential avoidance model. Behav Res Ther (2006) 44:371-94. doi: 10.1016/j.brat.2005.03.005

26. Hooley JM, Franklin JC. Why Do People Hurt Themselves? A New Conceptual Model of Nonsuicidal Self-Injury. Clin Psychol Sci (2017) 6:428-51. doi: 10.1177/2167702617745641
27. Taylor PJ, Jomar K, Dhingra K, Forrester R, Shahmalak U, Dickson JM. A meta-analysis of the prevalence of different functions of non-suicidal selfinjury. J Affect Disord (2018) 227:759-69. doi: 10.1016/j.jad.2017.11.073

28. Turner BJ, Jin HM, Anestis MD, Dixon-Gordon KL, Gratz KL. Personality pathology and intentional self-harm: cross-cutting insights from categorical and dimensional models. Curr Opin Psychol (2018) 21:55-9. doi: 10.1016/ j.copsyc.2017.09.009

29. Brown SA, Williams K, Collins A. Past and recent deliberate self-harm: emotion and coping strategy differences. J Clin Psychol (2007) 63:791-803. doi: $10.1002 /$ jclp. 20380

30. Armey MF, Crowther JH. A comparison of linear versus non-linear models of aversive self-awareness, dissociation, and non-suicidal self-injury among young adults. J Consult Clin Psychol (2008) 76:9-14. doi: 10.1037/0022-006x.76.1.9

31. Arens AM, Gaher RM, Simons JS. Child maltreatment and deliberate selfharm among college students: testing mediation and moderation models for impulsivity. Am J Orthopsychiatry (2012) 82:328-37. doi: 10.1111/j.19390025.2012.01165.x

32. Nicolai KA, Wielgus MD, Mezulis A. Identifying Risk for Self-Harm: Rumination and Negative Affectivity in the Prospective Prediction of Nonsuicidal Self-Injury. Suicide Life Threat Behav (2016) 46:223-33. doi: 10.1111/sltb.12186

33. Burke TA, Anne McArthur B, Daryanani I, Abramson LY, Alloy LB. Latent classes of trait affect and cognitive affective regulation strategies are associated with depression, non-suicidal self-injury, and well-being. J Affect Disord (2018) 225:180-7. doi: 10.1016/j.jad.2017.08.015

34. Bentley KH, Cassiello-Robbins CF, Vittorio L, Sauer-Zavala S, Barlow DH. The association between nonsuicidal self-injury and the emotional disorders: A meta-analytic review. Clin Psychol Rev (2015) 37:72-88. doi: 10.1016/ j.cpr.2015.02.006

35. Rodriguez-Blanco L, Carballo JJ, Baca-Garcia E. Use of Ecological Momentary Assessment (EMA) in Non-Suicidal Self-Injury (NSSI): A systematic review. Psychiatry Res (2018) 263:212-9. doi: 10.1016/j.psychres.2018.02.051

36. Muehlenkamp JJ, Engel SG, Wadeson A, Crosby RD, Wonderlich SA, Simonich $\mathrm{H}$, et al. Emotional states preceding and following acts of nonsuicidal self-injury in bulimia nervosa patients. Behav Res Ther (2009) 47:837. doi: $10.1016 /$ j.brat.2008.10.011

37. Andrewes HE, Hulbert C, Cotton SM, Betts J, Chanen AM. Ecological momentary assessment of nonsuicidal self-injury in youth with borderline personality disorder. Pers Disord (2017) 8:357-65. doi: 10.1037/per0000205

38. Armey MF, Crowther JH, Miller IW. Changes in ecological momentary assessment reported affect associated with episodes of nonsuicidal selfinjury. Behav Ther (2011) 42:579-88. doi: 10.1016/j.beth.2011.01.002

39. Houben M, Claes L, Vansteelandt K, Berens A, Sleuwaegen E, Kuppens P. The emotion regulation function of nonsuicidal self-injury: A momentary assessment study in inpatients with borderline personality disorder features. J Abnorm Psychol (2017) 126:89-95. doi: 10.1037/abn0000229

40. Kranzler A, Fehling KB, Lindqvist J, Brillante J, Yuan F, Gao X, et al. An Ecological Investigation of the Emotional Context Surrounding Nonsuicidal Self-Injurious Thoughts and Behaviors in Adolescents and Young Adults. Suicide Life Threat Behav (2018) 48:149-59. doi: 10.1111/sltb.12373

41. Hughes CD, King AM, Kranzler A, Fehling K, Miller A, Lindqvist J, et al. Anxious and Overwhelming Affects and Repetitive Negative Thinking as Ecological Predictors of Self-Injurious Thoughts and Behaviors. Cogn Ther Res (2019) 43:88-101. doi: 10.1007/s10608-019-09996-9

42. Nock MK, Prinstein MJ, Sterba SK. Revealing the Form and Function of SelfInjurious Thoughts and Behaviors: A Real-Time Ecological Assessment Study Among Adolescents and Young Adults. J Abnormal Psychol (2009) 118:81627. doi: $10.1037 / \mathrm{a} 0016948$

43. Klonsky ED. The functions of self-injury in young adults who cut themselves: Clarifying the evidence for affect-regulation. Psychiatry Res (2009) 166:260-8. doi: $10.1016 /$ j.psychres.2008.02.008

44. Claes L, Klonsky ED, Muehlenkamp J, Kuppens P, Vandereycken W. The affect-regulation function of nonsuicidal self-injury in eating-disordered patients: which affect states are regulated? Compr Psychiatry (2010) 51:38692. doi: 10.1016/j.comppsych.2009.09.001

45. O'Connor RC, Kirtley OJ. The integrated motivational-volitional model of suicidal behaviour. Philos Trans R Soc Lond B Biol Sci (2018) 373:20170268. doi: $10.1098 /$ rstb.2017.0268 
46. Klonsky ED, May AM. The Three-Step Theory (3ST): A New Theory of Suicide Rooted in the "Ideation-to-Action" Framework. Int $J$ Cogn Ther (2015) 8:114-219. doi: 10.1521/ijct.2015.8.2.114

47. Klonsky ED, Saffer BY, Bryan CJ. Ideation-to-action theories of suicide: a conceptual and empirical update. Curr Opin Psychol (2018) 22:38-43. doi: 10.1016/j.copsyc.2017.07.020

48. Victor SE, Scott LN, Stepp SD, Goldstein TR. I Want You to Want Me: Interpersonal Stress and Affective Experiences as Within-Person Predictors of Nonsuicidal Self-Injury and Suicide Urges in Daily Life. Suicide Life Threat Behav (2019) 49:1157-77. doi: 10.1111/sttb.12513.30159910

49. May AM, Klonsky ED. What Distinguishes Suicide Attempters From Suicide Ideators? A Meta-Analysis of Potential Factors. Clin Psychol: Sci Pract (2016) 23:5-20. doi: 10.1111/cpsp.12136

50. Khazem LR, Anestis MD. Thinking or doing? An examination of wellestablished suicide correlates within the ideation-to-action framework. Psychiatry Res (2016) 245:321-6. doi: 10.1016/j.psychres.2016.08.038

51. Nock MK, Hwang I, Sampson N, Kessler RC, Angermeyer M, Beautrais A, et al. Cross-national analysis of the associations among mental disorders and suicidal behavior: findings from the WHO World Mental Health Surveys. PLoS Med (2009) 6(8):e1000123. doi: 10.1371/journal.pmed.1000123

52. Hasking P, Rose A. A Preliminary Application of Social Cognitive Theory to Nonsuicidal Self-Injury. J Youth Adolesc (2016) 45:1560-74. doi: 10.1007/ s10964-016-0449-7

53. Dawkins JC, Hasking PA, Boyes ME, Greene D, Passchier C. Applying a cognitive-emotional model to nonsuicidal self-injury. Stress Health (2019) 35:39-48. doi: 10.1002/smi.2837

54. Epskamp S, van Borkulo CD, van der Veen DC, Servaas MN, Isvoranu AM, Riese H, et al. Personalized Network Modeling in Psychopathology: The Importance of Contemporaneous and Temporal Connections. Clin Psychol Sci (2018) 6:416-27. doi: 10.1177/2167702617744325

55. First MB, Williams JBW, Karg RS, Spitzer RL eds. Structured clinical interview for DSM-5-Research version (SCID-5 for DSM-5, research version; SCID-5$R V)$. Arlington, VA: American Psychiatric Association (2015).

56. Nock MK, Holmberg EB, Photos VI, Michel BD. Self-Injurious Thoughts and Behaviors Interview: development, reliability, and validity in an adolescent sample. Psychol Assess (2007) 19:309-17. doi: 10.1037/1040-3590.19.3.309

57. Watson D, Clark LA, Tellegen A. Devlopment and validation of brief measures of positive and negative affect: The PANAS scales. J Pers Soc Psychol (1988) 54:1063-70. doi: 10.1037/0022-3514.54.6.1063

58. Crawford JR, Henry JD. The positive and negative affect schedule (PANAS): construct validity, measurement properties and normative data in a large nonclinical sample. Br J Clin Psychol (2004) 43:245-65. doi: 10.1348/ 0144665031752934

59. Czyz EK, Bohnert AS, King CA, Price AM, Kleinberg F, Ilgen MA. Self-efficacy to avoid suicidal action: factor structure and convergent validity among adults in substance use disorder treatment. Suicide Life Threat Behav (2014) 44:698709. doi: 10.1111/sltb.12101

60. Henry JD, Crawford JR. The short-form version of the Depression Anxiety Stress Scales (DASS-21): construct validity and normative data in a large nonclinical sample. Br J Clin Psychol (2005) 44:227-39. doi: 10.1348/ 014466505X29657

61. Russell JA. Core affect and the psychological construction of emotion. Psychol Rev (2003) 110:145-72. doi: 10.1037/0033-295X.110.1.145

62. Shrout P, Lane SP. Psychometrics. In: Mehl MR, Conner TS, editors. Handbook of research methods for studying daily life. New York: Guilford Press (2012). p. 302-20.

63. Asparouhov T, Hamaker EL, Muthén B. Dynamic Structural Equation Models. Struct Equation Model: A Multidiscip J (2017) 25:359-88. doi: 10.1080/10705511.2017.1406803

64. Hamaker EL, Asparouhov T, Brose A, Schmiedek F, Muthen B. At the Frontiers of Modeling Intensive Longitudinal Data: Dynamic Structural Equation Models for the Affective Measurements from the COGITO Study. Multivariate Behav Res (2018) 53:820-41. doi: 10.1080/00273171.2018. 1446819
65. Asparouhov T, Muthén B. Comparison of models for the analysis of intensive longitudinal data. Struct Equation Model: A Multidiscip J (2019) 27:275-97. doi: $10.1080 / 10705511.2019 .1626733$

66. McNeish D, Hamaker EL. A primer on two-level dynamic structural equation models for intensive longitudinal data in Mplus. Psychol Methods (2019). doi: $10.1037 /$ met0000250

67. Glenn CR, Jaroszewski AC, Milner A, Kearns JC, Nock MK. Nonsuicidal selfinjury: Old problem, new disorder, limited data. In: Bromet E, editor. Longterm outcomes in psychopathology research: Rethinking the scientific agenda. New York: Oxford University Press (2015). p. 253-76.

68. Gilbert P, McEwan K, Mitra R, Franks L, Richter A, Rockliff H. Feeling safe and content: A specific affect regulation system? Relationship to depression, anxiety, stress, and self-criticism. J Positive Psychol (2008) 3:182-91. doi: 10.1080/17439760801999461

69. Fernandez-Aranda F, Jimenez-Murcia S, Santamaria JJ, Gunnard K, Soto A, Kalapanidas E, et al. Video games as a complementary therapy tool in mental disorders: PlayMancer, a European multicentre study. J Ment Health (2012) 21:364-74. doi: 10.3109/09638237.2012.664302

70. Paret C, Kluetsch R, Zaehringer J, Ruf M, Demirakca T, Bohus M, et al. Alterations of amygdala-prefrontal connectivity with real-time fMRI neurofeedback in BPD patients. Soc Cognit Affect Neurosci (2016) 11:95260. doi: $10.1093 /$ scan/nsw016

71. Fitzpatrick S, Kranzler A, Fehling K, Lindqvist J, Selby EA. Investigating the role of the intensity and duration of self-injury thoughts in self-injury with ecological momentary assessment. Psychiatry Res (2020). doi: 10.1016/ j.psychres.2020.112761

72. Armey MF. Ecological Momentary Assessment and Intervention in Nonsuicidal Self-Injury: A Novel Approach to Treatment. J Cogn Psychother (2012) 26:299-317. doi: 10.1891/0889-8391.26.4.299

73. Franklin JC, Fox KR, Franklin CR, Kleiman EM, Ribeiro JD, Jaroszewski AC, et al. A brief mobile app reduces nonsuicidal and suicidal self-injury: Evidence from three randomized controlled trials. J Consult Clin Psychol (2016) 84:54457. doi: $10.1037 /$ ccp0000093

74. Van Orden KA, Witte TK, Cukrowicz KC, Braithwaite SR, Selby EA, Joiner TEJr. The interpersonal theory of suicide. Psychol Rev (2010) 117:575-600. doi: $10.1037 / \mathrm{a} 0018697$

75. Joiner TE. Why people die by suicide. Cambridge: Harvard University Press (2005).

76. Allen NB, Nelson BW, Brent D, Auerbach RP. Short-term prediction of suicidal thoughts and behaviors in adolescents: Can recent developments in technology and computational science provide a breakthrough? J Affect Disord (2019) 250:163-9. doi: 10.1016/j.jad.2019.03.044

77. Kleiman EM, Miller A, Joyce VW, Nash CC, Buonopane RJ, Nock MK. Using Wearable Physiological Monitors With Suicidal Adolescent Inpatients: Feasibility and Acceptability Study. JMIR Mhealth Uhealth (2019) 7(9): e13725. doi: 10.2196/13725

78. Turner BJ, Baglole JS, Chapman AL, Gratz KL. Experiencing and Resisting Nonsuicidal Self-injury Thoughts and Urges in Everyday Life. Suicide Life Threat Behav (2019) 49:1332-46. doi: 10.1111/sltb.12510

79. Kleiman EM, Turner BJ, Fedor S, Beale EE, Picard RW, Huffman JC, et al. Digital phenotyping of suicidal thoughts. Depress Anxiety (2018) 35:601-8. doi: $10.1002 /$ da. 22730

Conflict of Interest: The authors declare that the research was conducted in the absence of any commercial or financial relationships that could be construed as a potential conflict of interest.

Copyright (๐ 2020 Kiekens, Hasking, Nock, Boyes, Kirtley, Bruffaerts, Myin-Germeys and Claes. This is an open-access article distributed under the terms of the Creative Commons Attribution License (CC BY). The use, distribution or reproduction in other forums is permitted, provided the original author(s) and the copyright owner(s) are credited and that the original publication in this journal is cited, in accordance with accepted academic practice. No use, distribution or reproduction is permitted which does not comply with these terms. 